\title{
Treatment of canine osteoarthritis with allogeneic platelet-rich plasma: review of five cases
}

\author{
José Catarino $^{1}$, Pedro Carvalho ${ }^{2,3}$, Sara Santos ${ }^{1}$, Ângela Martins ${ }^{1,4}$ and João Requicha ${ }^{1,5^{*}}$ \\ ${ }^{1}$ Faculty of Veterinary Medicine, University Lusófona, Lisbon, Portugal \\ ${ }^{2} C I V G$ - Vasco da Gama Research Center, University School Vasco da Gama, Campus Universitário, \\ Coimbra, Portugal \\ ${ }^{3}$ Vetherapy - Research and Development in Biotechnology, Coimbra, Portugal \\ ${ }^{4}$ Arrábida Veterinary Hospital and Functional Animal Rehabilitation Center, Azeitão, Portugal \\ ${ }^{5} C E C A V$ - Animal and Veterinary Research Center, Department of Veterinary Sciences, \\ University of Trás-os-Montes e Alto Douro, Vila Real, Portugal
}

\begin{abstract}
Background: Osteoarthritis (OA) is a major cause of chronic pain and lameness in dogs. Platelet-rich plasma (PRP) is a concentrate of growth and differentiation factors from the blood, which can be used in regenerative medicine strategies.

Aim: The main aim of this study was to evaluate the effect of allogeneic PRP on the treatment of canine OA.

Methods: Five dogs from several breeds, between 6 and 12 years old, and from both genders were studied. Clinical and imageological examinations diagnosed OA in the knee, tibiotarsal, elbow, and intercarpal joints. These dogs were refractory to medical therapy and to physical rehabilitation protocols that included shockwave therapy, laser therapy, electrostimulation, hydrotherapy, and diathermy.

Animals were treated with allogeneic PRP obtained from the blood of the five dogs, which was processed in a pool. Echoguided intra-articular PRP injection was administered under sedation and after aseptic field preparation. Lameness at walk and trot (five grades) and pain (five scores) were evaluated before treatment and 30, 60, and 90 days post-treatment.

Results: All animals presented improvements at 30 and 60 days in both parameters. Four dogs showed a decrease of three grades of lameness after 90 days and there was complete absence of lameness in 2 days. Pain was reduced from severe and moderate to mild in all the dogs after 30 days, and among them, three revealed no pain after 90 days.

Conclusion: This study sheds light on the applicability and safety of a single administration of allogeneic PRP in osteoarthritic dogs.
\end{abstract}

Keywords: Dog, Lameness, Osteoarthritis, Pain, Platelet-rich plasma..

\section{Introduction}

Osteoarthritis (OA) is a common chronic degenerative disease of synovial joints in both human and veterinary patients. The inflammatory process causes slow and progressive destruction of the subchondral bone, formation of osteophytes, thickening of the joint capsule, and synovitis (Malek et al., 2012; GlynJones et al., 2015). Ultimately, this condition leads to significant pain and associated lameness in companion animals, including dogs (Brown et al., 2007; Schaible, 2012).

At the present moment, there is no effective therapeutic protocol, either medical or surgical, that allows clinical resolution of OA (Bland, 2015). Therefore, the main focus includes pain control by using nonsteroidal anti-inflammatory drugs, opioid analgesics, as well as gabapentin or amantadine for chronic pain (Malek et al., 2012; KuKanich, 2013). Acupuncture is also referred to have a positive effect in pain management in veterinary patients as a part of a multimodal plan (Fry et al., 2014). This approach includes physical rehabilitation for the improvement of joint function and hindrance of the joint's degenerative processes (Alvarez et al., 2016). Weight reduction is referred to have a positive effect on the control of clinical signs of lameness in dogs with hip OA (Impellizeri et al., 2000). Dietary oral supplementation with chondroitin/glucosamine sulfate (McCarthy et al., 2007), omega-3 fish oil fatty acids (Fritsch et al., 2010), and beta-1,3/1,6-glucans (Beynen and Legerstee, 2010) are examples of adjuvant strategies to reduce clinical signs of OA and to prevent progression of the degenerative process. Other options include intraarticular injections of hyaluronan (Brandt et al., 2004) and intramuscular administration of polysulfated glycosaminoglycan (Fujiki et al., 2007).

Regenerative Medicine has seen a rapid evolution during recent years with new therapies based on the application of innovative biomaterials, adult stem cells,

*Corresponding Author: João Filipe Requicha. Department of Veterinary Sciences, University of Trás-os-Montes e Alto Douro, 
and different sources of growth and differentiation factors (GDF). One of the first-known regenerative approaches proposed in clinical practice was plateletrich plasma (PRP) (De Coppi, 2012; Guercio et al., 2012; Gato-Calvo et al., 2019).

PRP is a blood-derived biological product with a high concentration of platelets in a small volume of plasma. Methodologies to prepare PRP can rely on single centrifugation, double centrifugation, or selective blood-filtration procedures, and on manual or automatic systems operated in open or closed circuits (Gato-Calvo et al., 2019). After activation with calcium chloride, several GDFs are released from alpha granules stored inside platelets, which include plateletderived growth factor, transforming growth factor $\beta 1$, transforming growth factor $\beta 2$, growth factor vascular endothelial growth, fibroblast growth factor and epidermal growth factor, insulin-like growth factors 1 and 2, interleukin 8, keratinocyte growth factor, and connective tissue growth factor (Gnecchi et al., 2008; Alves et al., 2011; Cuervo et al., 2020). In addition, PRP also contains adhesion molecules, such as fibrinogen, fibronectin, and vitronectin (Sánchez-González et al., 2012). These bioactive molecules influence the processes of mitosis, chemotaxis, cell differentiation, growth of undifferentiated mesenchymal cells and the production of extracellular matrix, thus promoting tissue regeneration (Mata, 2013).

PRP can be categorized based on its composition as increased leukocytes with no activation, increased leukocytes with activation, minimal leukocytes with no activation, and minimal leukocytes with activation (Mishra et al., 2012). In order to have a therapeutic effect, the PRP should contain at least 1,000,000 platelets per microliter of plasma to provide the adequate release of GDF in the application site (Sánchez-González et al., 2012).

The therapeutic efficacy of PRP, either from commercial brands or in-house protocols, has been tested in vitro and in preclinical and clinical trials for oral and maxillofacial surgery, ophthalmology, dermatology, and musculoskeletal conditions in humans (GatoCalvo et al., 2019). In dogs, PRP has been used for the treatment of different osteoarticular diseases, including OA (Bland, 2015; Karayannopoulou et al., 2015; Vilar et al., 2018; Cuervo et al., 2020).
The aim of this study is to evaluate the effect of allogeneic PRP therapy on pain and joint function in dogs with $\mathrm{OA}$, and that are refractory to pharmacological and physical rehabilitation conventional approaches.

\section{Materials and Methods}

Study population and evaluated parameters

The studied series comprised five dogs treated at the Arrábida Veterinary Hospital with clinical and imageological diagnosis of OA. They were aged between 6 and 14 years, and two were Labrador Retrievers, one was a mixed breed dog, one was a German Shepherd, and one was a German Pointer (Table 1).

The orthopedic examination of the animals included the assessment of lameness grade and pain score on days $0,30,60$, and 90 after PRP application. Lameness was categorized from grade 1 to grade 5. Pain score was assessed by palpation and was categorized as follows: grade 0 , absence of pain and without clinical manifestation; grade 1, mild pain and the animal bends the limb, but does not vocalize or looks at the limb; grade 2, moderate pain and the animal bends the limb, does not vocalize, but looks at the limb; grade 3 , severe pain, the animal bends limb, vocalizes, and looks at limb; and finally, grade 4, severe pain, the animal pulls away the limb, vocalizes, shows aggressive behavior, and looks at limb.

\section{Therapeutic approach before PRP therapy}

Prior to the study, the dogs were subjected to multidisciplinary protocols for pain management and to improve gait biomechanical function. Pharmacological treatment included meloxicam $(0.1 \mathrm{mg} / \mathrm{kg}$, per os, SID) for $8-15$ days used in acute pain episodes, tramadol (2-4 mg/kg, per os, BID or TID), and gabapentin (5 $\mathrm{mg} / \mathrm{kg}$, per os, BID or TID) for chronic pain control. The animals were supplemented with chondroitin and glucosamine sulfate orally and no specific medical diet was prescribed.

In addition to the referred medical treatment, animal 1 was subject to shockwave therapy using the equipment PulseVet, Alpharetta, GA, USA; animals 3 and 4 were prescribed laser therapy (Lite Cure, New Castle, DE), electrostimulation (BTL 4000 Premium, Famões, Portugal), and hydrotherapy (Hidro Physio, Shropshire, United Kingdom); and animal five was treated with diathermy therapy (BTL 6000, Famões, Portugal) (Fig. 1).

Table 1. Summary of cases included in this study regarding the breed, age, and affected joint.

\begin{tabular}{clcl}
\hline Animal & Breed & Age (years) & Joint \\
\hline 1 & Mixed breed & 12 & Knee \\
2 & Labrador Retriever & 10 & Tibiotarsal \\
3 & Labrador Retriever & 13 & Knee \\
4 & German Shepherd & 14 & Elbow \\
5 & German Pointer & 6 & Carpal \\
\hline
\end{tabular}


After therapy, the animals did not reveal any clinical improvement and, during a 6-months period, they had not undergone any pharmacological or physical rehabilitation procedures.

\section{PRP therapy}

The osteoarthritic joints of the five studied animals were targeted for allogeneic PRP therapy. The PRP gel was provided by Vetherapy (Coimbra, Portugal). In order to obtain PRP, venous blood was collected in a 250-ml transfusion bag with citrate phosphate dextrose. The blood samples were sent to the laboratory and were analyzed to make sure animals were vaccinated. Within 12 hours, the blood samples were processed under Vetherapy's proprietary protocol by the dual centrifugation technique (one low-speed and one high-speed centrifugation) with the standardization of platelet counts per $\mathrm{ml}$ (minimum of 1 million platelets per microliter). The obtained PRP was pooled together, stored and frozen, and sent to Arrábida Veterinary Hospital in 1.5-ml vials on dry ice. In all the dogs from which blood was obtained using an allogeneic approach, echoguided intraarticular PRP injection was administered under sedation with a combination of $0.2 \mathrm{mg} / \mathrm{kg}$ of midazolam, $0.2 \mathrm{mg} / \mathrm{kg}$ of methadone, and $0.6 \mathrm{mg} /$ $\mathrm{kg}$ of propofol, intravenously and after aseptic field preparation (Fig. 2). After this procedure, the animals were evaluated after 30, 60, and 90 days.

\section{Statistical analysis}

The data obtained were subject to descriptive statistical analysis that included the calculation of absolute and relative frequencies, means, and standard deviation using the Microsoft Office Excel 2013 software (Microsoft, United States of America).

\section{Ethical approval}

There were no ethical issues to address.

\section{Results}

It can be seen that all animals improved after 90 days of treatment. In this scenario, two clinical parameters were evaluated: the grade of lameness on days 0,30 , 60 , and 90 (Table 2 ) and the score of pain on days 0,30 , 60, and 90 (Table 3).

All animals showed a decrease in lameness grade after 90 days. Animal 2, after day 90, presented a normal gait. The two animals with grade III lameness, after 90 days of PRP administration, improved for grades I and II, respectively. The two animals that presented grade II lameness evolved to grade 0 , i.e., without lameness. The only animal with grade IV lameness, after PRP application decreased to a grade II.

For pain evaluation, there were positive results, with the first three animals becoming painless, while animals 4 and 5 went from having severe and moderate pain to mild pain after day 90 , respectively.
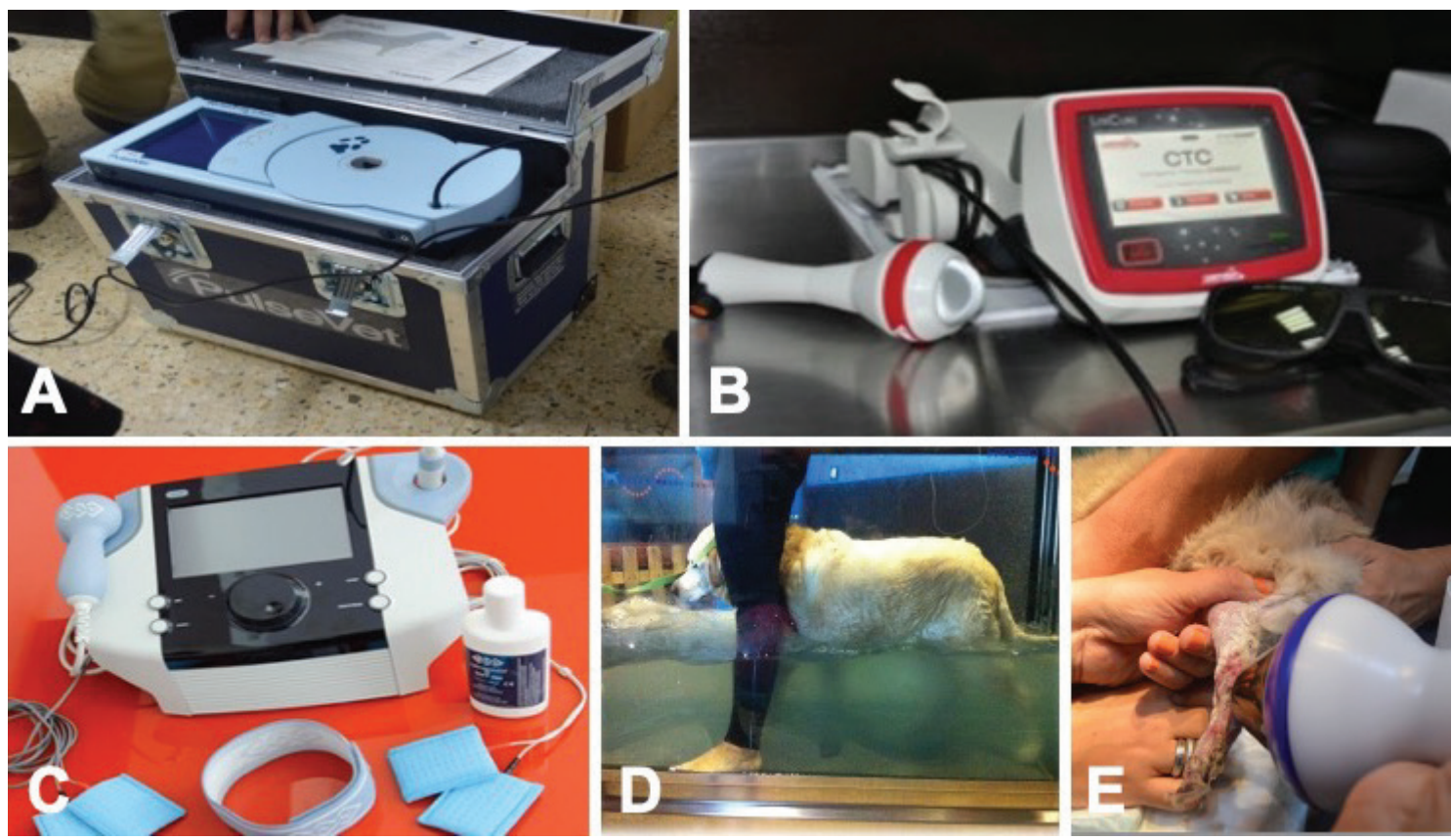

Fig. 1. Equipment used in the physical rehabilitation protocol. (A) Extracorporeal shockwaves. (B) Class IV laser therapy. (C) Electrostimulation therapy. (D) Hydrotherapy. (E) Diathermy therapy. 


\section{Discussion}

One of the purposes of using therapies, such as PRP, is to enhance healing and stimulate the regeneration of injured tissues that have a weak natural regenerative capacity, such as tendons, ligaments, menisci, and cartilages (Gutierrez-Nibeyro, 2011). It is believed that platelets can mediate the release of high concentrations of growth factors. It is described that these growth factors can favorably induce analgesia, soft tissue healing, regulation of anti-inflammatory signals and vascularization, and innervation of autografts, making

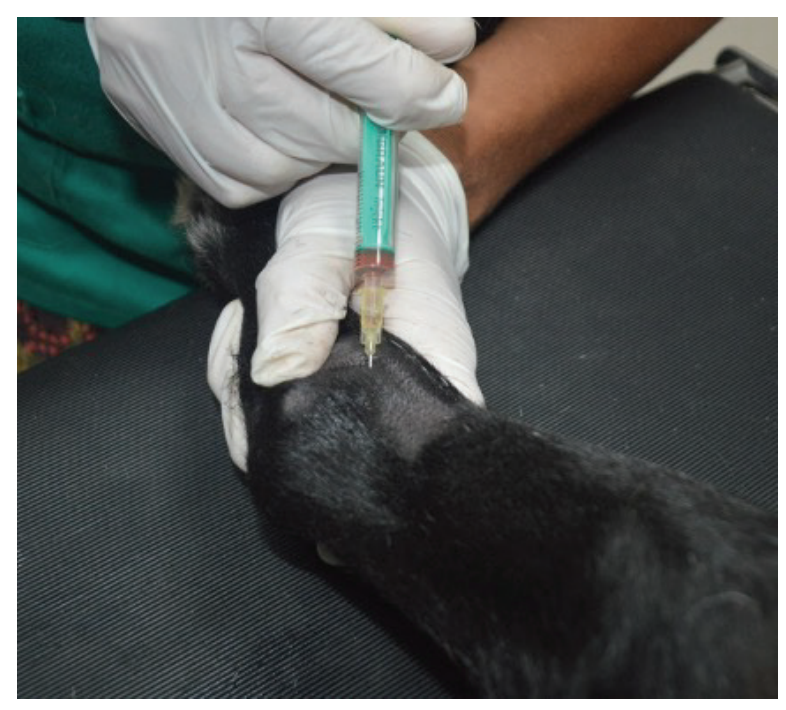

Fig. 2. Intraarticular injection of PRP after echoguided localization of the articular cavity.
PRP an interesting therapeutic approach to OA (Vilar et al., 2018)

In this work, our study population comprised mainly adult to geriatric large breed dogs (Labrador Retriever, German Shepherd, and German Pointer), described as predisposed to the development of OA (Bland, 2015). The clinical results obtained were promising because PRP administration was well tolerated by these types of patients and clinical improvement was documented. These results are in agreement with the published scientific literature.

A recent study on dogs showed promising results on the management of OA caused by cruciate ligament rupture using minimal leukocyte PRP. The studied population demonstrated an increase in many different gait associated parameters, such as peak vertical forces, angular range of motion, and stance time without associated side effects (Vilar et al., 2018). Studies on horses have shown that intralesional treatment of PRP promoted resolution of tendinitis associated with decreased lameness, edema, local sensitivity, as well as improved ultrasound image of evaluated structures (Carmona et al., 2007). Abellanet and Prades (2009) studied 42 sport horses with OA lesions, wherein 70\% of horses receiving PRP therapy resumed their normal work routine and only $9.5 \%$ of the animals relapsed when compared to the control group, wherein 33\% of the horses relapsed. In the latter two studies, no local or systemic side effects were reported following PRP treatment (Carmona et al., 2007; Abellanet and Prades, 2009).

The combined application of PRP with stem cells, such as adipose-derived stem cells (ASCs), in osteoarticular diseases in dogs has also demonstrated promising

Table 2. Lameness grades at day 0 and at days 30, 60, and 90 after PRP administration.

\begin{tabular}{ccccc}
\hline Animal & Day 0 & Day 30 & Day 60 & Day 90 \\
\hline 1 & Grade III & Grade II & Grade II & Grade I \\
2 & Grade II & Grade II & Grade I & No lameness \\
3 & Grade IV & Grade III & Grade II & Grade II \\
4 & Grade III & Grade III & Grade II & Grade II \\
5 & Grade II & Grade II & Grade I & No lameness \\
\hline
\end{tabular}

Table 3. Pain scores at day 0 and at days 30, 60, and 90 after PRP administration.

\begin{tabular}{ccccc}
\hline Animal & Day 0 & Day 30 & Day 60 & Day 90 \\
\hline 1 & Intense & Moderate & Mild & Painless \\
2 & Moderate & Mild & Mild & Painless \\
3 & Intense & Moderate & Mild & Painless \\
4 & Severe & Intense & Mild & Mild \\
5 & Moderate & Mild & Mild & Mild \\
\hline
\end{tabular}


results for its significant effectiveness in improving animal welfare during the recovery period. Although a relatively new concept, the strategy is appealing because the resulting regenerative matrix delivers a potent trilogy of undifferentiated cells, fibrin matrix, and differentiation factors (Sánchez-González et al., 2012). The concomitant application of ASCs and bone marrow is considered as one of the most promising therapies (De Coppi, 2012). ASC-based therapies have regenerative potential because of their ability to differentiate into damaged tissue cells and the ability to chemically recruit other undifferentiated cells from the recipient organism to the site of tissue regeneration. In addition, they are immunoprivileged cells, they do not trigger a rejection reaction by the body, they have a very beneficial immunomodulatory effect on the treatment of inflammatory and immune-mediated diseases (Requicha et al., 2016) and are a useful alternative to prolonged glucocorticoid and non-steroidal antiinflammatories. These drugs can induce nefarious side effects, such as immunosuppression, iatrogenic hyperadrenocorticism, gastrointestinal ulceration, and renal or hepatic failure (Johnston and Budsberg, 1997) The use of PRP in humans for OA treatment is still a bit controversial and lacks standardization (Gato-Calvo et al., 2019). For authors, this can be seen as an opportunity for further comparative studies in order to contribute to a better understanding of this type of therapeutics in companion animals and helping with standardization and future translation to human applications.

\section{Conclusion}

PRP has therapeutic applicability in different animal species, resulting in a decrease of clinical signs associated with osteoarticular lesions.

At the end of the treatment, clinical evaluation showed that, in some cases, there were no signs of joint pain in the treated joints, as well as no lameness. The encouraging results of this study indicate the need for more comprehensive studies with larger numbers of animals in order to validate the method, aiming for it to become commonly administered to companion animals with degenerative joint disease.

Allogeneic administration of PRP has consistently been shown to be a simple, rapid, cost-effective, and safe way to assist the treatment of this disease. It is believed that the therapeutic use of this procedure can help in obtaining clinical improvement of diseased animals, which results in better quality of life and animal welfare.

\section{Acknowledgments}

This work is funded by the national funds through the Foundation for Science and Technology (FCT), I.P., under the Institutional Call to Scientific Employment Stimulus CEECINS/00127/2018, and supported by the project UIDB/CVT/00772/2020.

\section{Conflict of interest}

The authors declare that there is no conflict of interest. $\mathrm{PC}$ is the founder and CEO of Vetherapy who provided the PRPs for this study but had no involvement in the PRP administration and monitoring of the animals throughout the study.

\section{Authors contributions}

JR conceived the idea; JR and PC designed the study; AM was responsible for the clinical cases; JC, AM, and SS analyzed the data; JC, PC, and JR wrote the paper with input from all the authors.

\section{References}

Abellanet, I. and Prades, M. Intraarticular platelet rich plasma (PRP) therapy: evaluation in 42 sport horses with OA. Proceedings of the 11th International Congress of the World Equine Veterinary Association, Guarujá, Brazil, 2009. Available via http://www.ivis.org/proceedings/weva/2009/72. pdf?:LA=1

Alvarez, L.X., Fox, P.R., Van-Dyke, J.B. and Grigsby, P. 2016. Survey of referring veterinarians' perceptions of and reasons for referring patients to rehabilitation facilities. J. Am. Vet. Med. Assoc. 249, 807-813.

Alves, A., Stewart, A., Dudhia, J., Kasashima, Y., Goodship, A.E. and Smith, R.K.W. 2011. Cellbased therapies for tendon and ligament injuries. Vet. Clin. North Am. Equine Pract. 27(2), 315-333.

Beynen, A.C. and Legerstee, E. 2010. Influence of dietary beta-1,3/1,6-glucans on clinical signs of canine osteoarthritis in a double-blind, placebocontrolled trial. Am. J. Anim. Vet. Sci. 5(2), 97-101.

Bland, S.D. 2015. Canine osteoarthritis and treatments: a review. Vet. Sci. Dev. 5(2), 84-89.

Brandt, K.D., Smith, G.N. and Myers, S.L. 2004. Hyaluronan injection affects neither osteoarthritis progression nor loading of the OA knee in dogs. Biorheol. 41(3-4), 493-502.

Brown, D., Boston, C., Coyne, J. and Farrar, J. 2007. Development and psychometric testing of an instrument designed to measure chronic pain in dogs with osteoarthritis. Am. J. Vet. Res. 8(6), 631637.

Carmona, J., Argüelles, D., Climent, F. and Prades, M. 2007. Autologous platelet concentrates as a treatment of horses with osteoarthritis: a preliminary pilot clinical study. J. Equine Vet. Sci. 27(4), 167-170.

Cuervo, B., Rubio, M., Chicharro, D., Damiá, E., Santana, A., Carrillo, J.M., Romero, A.D., Vilar, J.M., Cerón, J.J. and Sopena, J.J. 2020. Objective comparison between platelet rich plasma alone and in combination with physical therapy in dogs with osteoarthritis caused by hip dysplasia. Animals 10(2), E175. 
De Coppi , P. 2012. Regeneration from fat: a clinical reality? Stem Cells Transl. Med. 1(3), x; doi:10.1002/sctm.2012.1.3.x

Fritsch, D.A., Allen, T.A., Dodd, C.E., Jewell, D.E., Sixby, K.A., Leventhal, P.S., Brejda, J. and Hahn, K.A. 2010. A multicenter study of the effect of dietary supplementation with fish oil omega-3 fatty acids on carprofen dosage in dogs with osteoarthritis. J. Am. Vet. Med. Assoc. 236(5), 535-539.

Fry, L.M., Neary, S.M., Sharrock, J. and Rychel, J.K. 2014. Acupuncture for analgesia in veterinary medicine. Top. Companion Anim. Med. 29(2), 35-42.

Fujiki, M., Shineha, J., Yamanokuchi, K., Misumi, K. and Sakamoto, H. 2007. Effects of treatment with polysulfated glycosaminoglycan on serum cartilage oligomeric matrix protein and C-reactive protein concentrations, serum matrix metalloproteinase-2 and -9 activities, and lameness in dogs with osteoarthritis. Am. J. Vet. Res. 68(8), 827-833.

Gato-Calvo, L., Magalhães, J., Ruiz-Romero, C., Blanco, F.J. and Burguera, E.F. 2019. Platelet-rich plasma in osteoarthritis treatment: review of current evidence. Ther. Adv. Chronic Dis. 10, 1-18.

Glyn-Jones, S., Palmer, A.J., Agricola, R., Price, A.J., Vincent, T.L., Weinans, H. and Carr, A.J. 2015. Osteoarthritis. Lancet 386(9991), 376-387.

Gnecchi, M., Zhang, Z., Ni, A. and Dzau, V. 2008. Paracrine mechanisms in adult stem cell signaling and therapy. Circ. Res. 103(11), 1204-1219.

Guercio, A., Marco, P., Casella, S., Cannella, V., Russotto, L., Purpari, G., Bella, S. and Piccione, G. 2012. Production of canine mesenchymal stem cells from adipose tissue and their application in dogs with chronic osteoarthritis of the humeroradial joints. Cell Biol. Int. 36(2), 189-194.

Gutierrez-Nibeyro, S.D. 2011. Commercial cell-based therapies for musculoskeletal injuries in horses. Vet. Clin. North Am. Equine Pract. 27(2), 363-371.

Impellizeri, J.A., Tetrick, M.A. and Muir, P. 2000. Effect of weight reduction on clinical signs of lameness in dogs with hip osteoarthritis. J. Am. Vet. Med. Assoc. 216(7), 1089-1091.

Johnston, S.A. and Budsberg, S.C. 1997. Nonsteroidal anti-inflammatory drugs and corticosteroids for the management of canine osteoarthritis. Vet. Clin. North Am. Small Anim. Pract. 27(4), 841-862.
Karayannopoulou, M., Psalla, D. and Kazakos, G. 2015. Effect of locally injected autologous plateletrich plasma on second intention wound healing of acute full thickness skin defects in dogs. Vet. Comp. Orthop. Traumatol. 3, 172-178.

KuKanich, B. 2013. Outpatient oral analgesics in dogs and cats beyond nonsteroidal antiinflammatory drugs: an evidence-based approach. Vet. Clin. North Am. Small Anim. Pract. 43(5), 1109-1125.

Malek, S., Sample, S.J., Schwartz, Z., Nemke, B., Jacobson, P.B., Cozzi, E.M., Schaefer, S.L., Bleedorn, J.A., Holzman, G. and Muir, P. 2012. Effect of analgesic therapy on clinical outcome measures in a randomized controlled trial using client-owned dogs with hip osteoarthritis. BMC Vet. Res. 8, 185.

Mata, J. 2013. Platelet rich plasma. A new treatment tool for the rheumatologist? Reumatol. Clin. 9(3), 166-171.

McCarthy, G., O'Donovan, J., Jones, B., McAllister, H., Seed, M. and Mooney, C. 2007. Randomised double-blind, positive controlled trial to assess the efficacy of glucosamine/chondroitin sulfate for the treatment of dogs with osteoarthritis. Vet. J. 174(1), 54-61.

Mishra, A., Harmon, K., Woodall, J. and Vieira, A. 2012. Sports medicine applications of platelet rich plasma. Curr. Pharm. Biotechnol. 13, 1185-1195.

Requicha, J.F., Viegas, C.A., Hede, S., Leonor, I.B., Reis, R.L. and Gomes, M.E. 2016. Design and characterization of a biodegradable double-layer scaffold aimed at periodontal tissue-engineering applications. J. Tissue Eng. Regen. Med. 10(5), 392-403.

Sánchez-González, D., Méndez-Bolaina, E. and TrejoBahena, N. 2012. Platelet-rich plasma peptides: key for regeneration. Int. J. Pept. 2012, 10.

Schaible, H.G. 2012. Mechanisms of chronic pain in osteoarthritis. Curr. Rheumatol. Rep. 14(6), 549556.

Vilar, J., Manera, M.E., Santana, A., Spinella, G., Rodriguez, O., Rubio, M., Carrillo, J.M., Sopena, J. and Batista, M. 2018. Effect of leukocyte-reduced platelet-rich plasma on osteoarthritis caused by cranial cruciate ligament rupture: a canine gait analysis model. PLoS One 13(3), e0194752. 